\title{
Title: Nickel-Catalyzed 1,2-Carboamination of Alkenyl Alcohols
}

Authors: Taeho Kang ${ }^{1}$, Nana Kim${ }^{1}$, Peter T. Cheng ${ }^{2}$, Hao Zhang ${ }^{2}$, Klement Foo ${ }^{2}$, and Keary M. Engle ${ }^{1 *}$

\begin{abstract}
An alcohol-directed, nickel-catalyzed three-component umpolung carboamination of unactivated alkenes with aryl/alkenylboronic esters and electrophilic aminating reagents is reported. This transformation is enabled by specifically tailored $O-(2,6-$ dimethoxybenzoyl)hydroxylamine electrophiles that suppress competitive processes, including undesired $\beta$-hydride elimination and transesterification between the alcohol substrate and electrophile. The reaction delivers the desired 1,2-carboaminated products with excellent regioand diastereoselectivity and exhibits a broad scope of coupling partners and alkenes, including complex natural products. Various mechanistic experiments and analysis of the stereochemical outcome with a cyclic alkene substrate, as confirmed by X-ray crystallographic analysis, support alcohol-directed syn-insertion of an organonickel(I) species.
\end{abstract}

\section{Introduction.}

Substituted aliphatic amines are substructures with essential biological importance in medicines, agrochemicals, and natural products ${ }^{1,2}$. Thus, the invention of methods to prepare such prized motifs from readily available starting materials in a manner that is rapid, selective, and modular is a captivating pursuit. In this context, alkene carboamination, in which a nitrogen substituent and a carbon substituent are added across a $\mathrm{C}=\mathrm{C}$ bond, has been actively pursued as a strategy with great promise in organic synthesis (Figure 1A). Early pioneering examples of alkene carboamination centered on various two-component strategies (Figure 1B). This includes use of carbon/nitrogen 
functional groups tethered to the alkene of interest. For example, Wolfe and others have developed carboamination reactions using intramolecular azacyclization of nitrogen-tethered alkenes under palladium or nickel catalysis, which enables facile preparation of various azaheterocycles ${ }^{3-6}$. Additionally, several groups independently demonstrated heteroannulations in which alkenes are coupled with bifunctional coupling partners using different transition metal catalysts ${ }^{7-11}$. Moreover, use of ambiphilic reagents possessing activatable heteroatom-heteroatom bonds has also enabled carboamination of electronically activated alkenes or 1,3-dienes ${ }^{12,13}$.

Despite these remarkable achievements, two-component alkene carboamination possesses inherent limitations in terms of modularity and structural diversity since two of three putative reaction components must be colocalized on the same molecule. Moreover, existing methods generally require use of non-basic amine coupling partners that are less likely to cause catalyst poisoning, such as sulfonamides, amides, carbamates, and anilines, which limits direct access to valuable aliphatic amine products. To address these shortcomings, we and others have endeavored to develop three-component carboamination reactions using various mechanistic paradigms. Such transformations have historically been fraught with issues, including competitive two-component coupling, difficulty in controlling regioselectivity, and formation of undesired side products that arise from unstable alkyl-metal or alkyl radical intermediates. Thus, three-component carboamination has been limited to conjugated alkenes ${ }^{14,15}$, strained cyclopropenes ${ }^{16,17}$ or alkenes bearing directing auxiliary groups ${ }^{18,19}$. For instance, in 2019 our lab developed a nickel-catalyzed umpolung 1,2-carboamination that unites alkenes bearing the strongly coordinating 8aminoquinoline (AQ) directing auxiliary with electrophilic aminating reagents and organozinc nucleophiles. Although this system offered high regio- and stereoselectivity, the method has an intrinsic limitation in requiring two concession steps for auxiliary installation and removal. In 
addition, the use of air- and moisture-sensitive organozinc reagents, which are less readily available compared with bench-stable alternatives, also diminishes the synthetic flexibility and practical utility of the method.

To invent a next-generation carboamination system that overcomes these obstacles, we sought to employing common, readily available alkene starting materials containing only native chemical functionality in conjunction with bench-stable coupling partners. Specifically, we were attracted to alkenyl alcohol substrates because these bear the most common, readily available, and synthetically versatile functional group, the hydroxyl group. Employing a hydroxyl group as a directing group in late-transition metal catalysis for reactions beyond hydrogenation is challenging for a number of interrelated reasons. First, coordination of the hydroxyl group with late-transition metals is relatively weak, meaning the resultant alkyl metallacycle intermediate may possess dynamic coordination chemistry and conformational flexibility, opening the possibility for side reactions, such as $\beta-\mathrm{H}$ elimination ${ }^{20}$. In addition, the innate nucleophilicity of alcohols (or metal alkoxides) can result in direct nucleophilic attack on the electrophilic coupling partners or cyclization onto the pendant alkene, interfering with the desired transformation. Moreover, many late transition metals are known to oxidize alcohols to the corresponding aldehydes or ketones ${ }^{21}$. For these reasons, seemingly straightforward extension of late-transition-metal-catalyzed alkene functionalization reactions directed by protected amines or carbonyl groups ${ }^{22-30}$ to alkenyl alcohol substrates is generally not possible without introduction of a protecting group or directing auxiliary $^{31}$. Indeed, free-alcohol-directed alkene functionalizations have generally been limited to oxophilic early transition metal catalysts, with the most notable success in epoxidation ${ }^{32,33}$. Using stoichiometric titanium with catalytic chiral ligand, Burns and co-workers have recently achieved three-component dihalogenations and haloazidation reactions of alkenes ${ }^{34-36}$. To the best of our 
knowledge, alcohol-directed metal-catalyzed three-component alkene difunctionalization has not yet been demonstrated. Herein, we describe the catalytic three-component coupling of alkenyl alcohols, various aliphatic amine electrophiles, and organoboronic acids enabled by the combined use of nickel catalysis and fine-tuned nitrogen activating groups (Figure 1C).

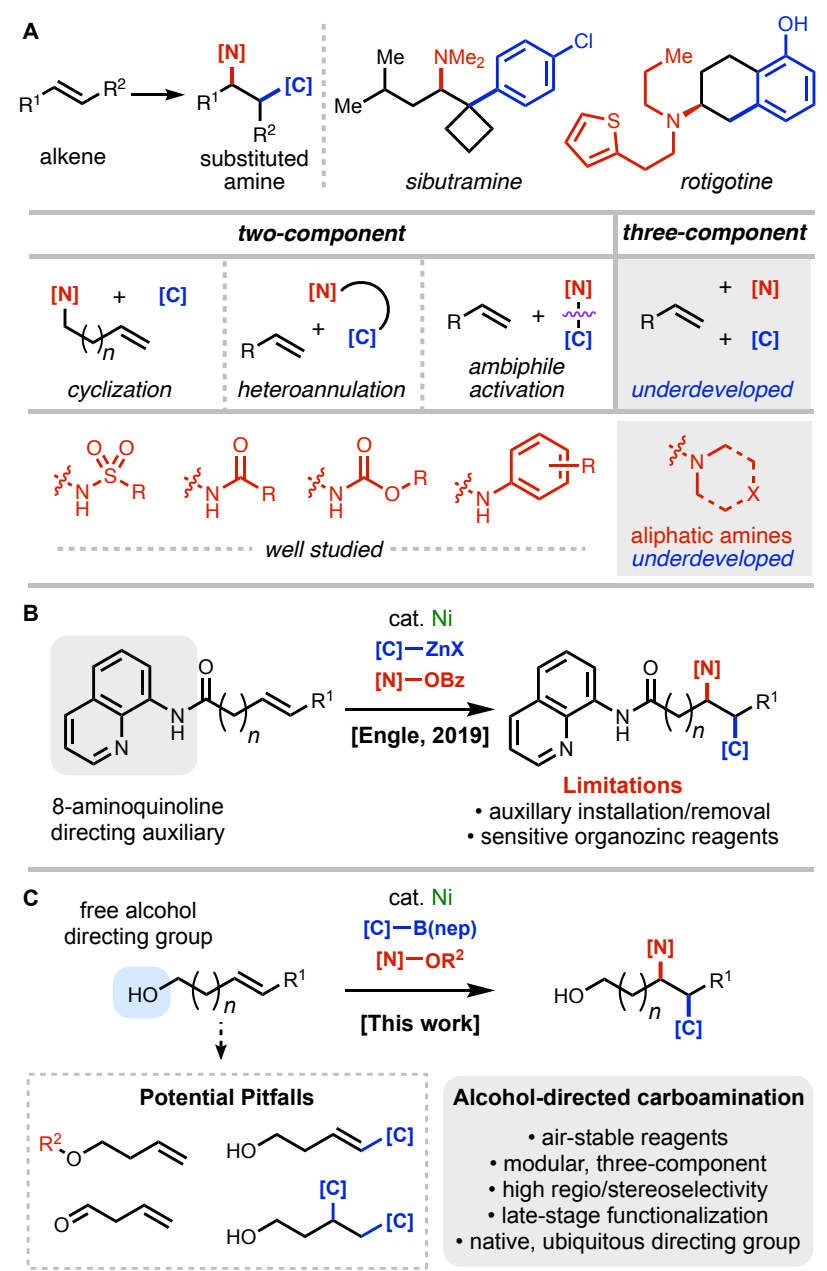

Fig. 1 Summary of previous and current work. A. Carboamination of alkenes and structures related commercial drugs. B. Previous nickel-catalyzed auxiliary-directed alkene carboamination (Ref. 19). C. Alcohol-directed 1,2-carboamination of unactivated alkenes developed in this study. 


\section{Results and Discussion.}

Optimization. We initiated our study with 3-buten-1-ol (1a) as the model substrate, $O$-benzoyl hydroxylmorpholine (2a) as an electrophilic aminating reagent ${ }^{19}$, and $\mathrm{Ni}(\operatorname{cod})_{2}$ as the precatalyst (Table 1). In place of air- and moisture-sensitive organozinc nucleophiles, we opted for PhB(nep) as the standard bench-stable nucleophilic coupling partner. After initial exploratory screening, we were able to observe the desired three-component conjunctive cross-coupling product in $17 \%$ yield with $t$ - $\mathrm{BuOH}$ as solvent and $\mathrm{KO} t$ - $\mathrm{Bu}$ as base. In addition to the desired product, we detected three major side products: 1) styrenyl alcohol product 4, likely arising from an oxidative Heck pathway, 2) benzoyl ester product 5 from transesterification between the starting material 1a and the amine electrophile $\mathbf{2 a}$, and 3 ) small amounts (5-10\% by $\left.{ }^{1} \mathrm{H} \mathrm{NMR}\right)$ of the hydroarylation product of $\mathbf{1 a}$, 4-phenylbutanol. We envisioned that the yield of the desired 1,2-arylamination product could be improved by suppressing these side products through the use of sterically and electronically tuned $\mathrm{N}-\mathrm{O}$ reagents. Introducing a bulky $O$-pivaloyl group on the hydoxylamine (2b) successfully inhibited transesterification with the alcohol starting material and increased the desired product yields, though the Heck byproduct 4 was still present. Furthermore, we observed a general trend that more electron-rich benzoyl groups on the electrophile delivered higher yields of desired product $(\mathbf{2 c}-\mathbf{2 e})$. Based on these two observations, we expected that electron-donating groups at the ortho-positions of the electrophile would enhance the yield of the desired product by suppressing undesired side product formation both sterically and electronically. Interestingly, use of a sterically bulkier mesityl group on the electrophile (2g) gave higher yield of desired product along with only trace amounts of both side products. In addition, more electron-donating substituents on the benzoyl electrophiles (2h and $\mathbf{2 i}$ ) further increased the product yield. A control experiment with $\mathbf{2 f}$ which has 2,6-disubstitution but possesses fluoro substituents with electron- 
withdrawing character revealed the importance of both the substitution pattern and electronic properties of the benzoyl activating group. The 2,6-dimethoxylbenzoyl activating group (as in $\mathbf{2 h}$ ) was ultimately selected for further study due to its comparative ease of installation. Notably, the Buchwald group has previously documented the benefits of using electron-rich benzoyl activating groups for accelerating $\mathrm{L}_{n} \mathrm{CuH}$ regeneration in copper-catalyzed umpolung alkene hydroamination. ${ }^{37}$ Our data expand upon these earlier findings to encompass alternative catalytic manifolds, demonstrating that precisely tailored activating groups on the electrophile can not only increase product yield but also allow for control of pathway selectivity (i.e., product distribution) in a complex multi-component nickel-catalyzed reaction system (vide infra).

The reaction was further evaluated under different conditions. Corresponding $\mathrm{PhB}(\mathrm{OH})_{2}$ and $\mathrm{PhB}(\mathrm{pin})$ nucleophiles also delivered the desired product, albeit in lower yields than standard $\mathrm{PhB}$ (nep) (Entry 1). Lower (room temperature) or higher temperature $\left(70^{\circ} \mathrm{C}\right)$ did not improve the product yield (Entries 2). We found that lower catalyst loading (5 mol\%) also gave an excellent yield, but higher catalyst loading ( $25 \mathrm{~mol} \%$ ) did not further increase the yield (Entry 3 ). In addition, the weaker base potassium hydroxide was found to be incompatible, while tert-butoxide salts with varying countercations were nearly equally effective (Entries 4 and 5). Importantly, the benchstable precatalyst, $\mathrm{NiBr}_{2} \cdot$ glyme, could be used in place of $\mathrm{Ni}(\mathrm{COD})_{2}$ allowing the reaction to be performed without an inert atmosphere glovebox with only slightly diminished yield (Entry 7, see SI for additional details). 


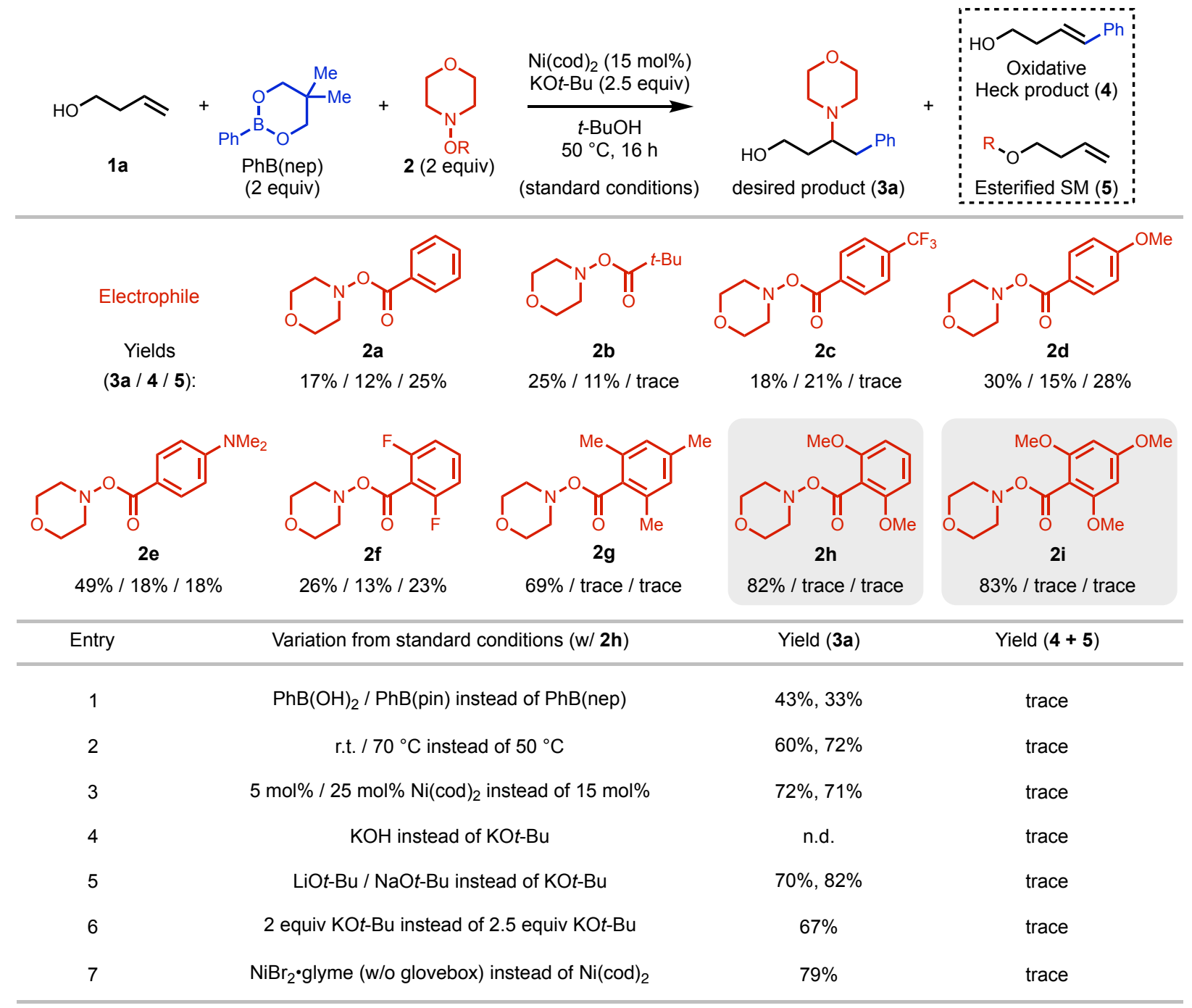

Table 1. Optimization of reaction. Reaction conditions: 1a ( $0.1 \mathrm{mmol}), \mathrm{PhB}(\mathrm{nep})(0.2 \mathrm{mmol}), 2$ (0.2 mmol), $\mathrm{KO} t$-Bu $(0.25 \mathrm{mmol}), \mathrm{Ni}(\operatorname{cod})_{2}(0.015 \mathrm{mmol}), t-\mathrm{BuOH}(1 \mathrm{~mL}), 50{ }^{\circ} \mathrm{C}, 16 \mathrm{~h}$. Percentages represent ${ }^{1} \mathrm{H}$ NMR yields using 1,3,5-trimethoxybenzene as internal standard; n.d. = not detected.

Nucleophile and electrophile scope. Having identified the optimal reaction conditions, we next examined the nucleophilic coupling partners. Arylboronic ester nucleophiles bearing electrondonating substituents in the para position reacted in excellent to good yields (3b-3d). In addition, 
fluoride, chloride, and protected amine substituents were compatible with the reaction conditions $(\mathbf{3 e}-\mathbf{3 g})$. On the other hand, arylboronic ester nucleophiles bearing electron-withdrawing groups in the para position resulted in lower yields, presumably due to less favorable migratory insertion of the carbon nucleophiles $(\mathbf{3 h}-\mathbf{3 j})$. Several ortho- and meta-substituted aryl nucleophiles also gave the desired product in moderate yields $(\mathbf{3 k}-\mathbf{3 p})$. Gratifyingly, alkenylboronic ester nucleophiles worked well to yield homologated alcohol products with only slightly lower yields than those of aryl nucleophiles (3q-3u). On the other hand, alkyl-, highly electron-deficient aryl-, and heterocycle-containing $\mathrm{B}(\mathrm{nep})$ coupling partners were ineffective under the reaction conditions (see SI for details).

We next explored the nitrogen electrophile scope. Medicinally relevant 6-membered azaheterocycles, including piperidine, $N$-Boc-protected piperazine, and thiomorpholine derived electrophiles gave excellent to moderate yields (3v-3x). Additionally, various substituted cyclic amines also delivered the corresponding products in excellent to synthetically viable yields $(\mathbf{3 y}-$ 3ac). Notably, product $\mathbf{3 z}$ could be used as a primary amine surrogate through simple deprotection, and a commercial drug (paroxetine) derived electrophile was also compatible with the reaction conditions (3ac). Moreover, 5- and 7-membered cyclic amine electrophiles were also successful, although with diminished yields (3ad-3ag). We also investigated the acyclic amine scope and observed attenuated reactivity, especially for bulky acyclic amine electrophiles. Indeed, dimethylamine electrophile gave a moderate yield, but slightly bulkier methylallylamine and diethylamine electrophiles showed low yields (3ah-3aj). In addition, a secondary amine, $N$-Bocprotected amine, imines, electron-deficient phthalimide, and other heterocyclic electrophiles did not afford desired product under the reaction conditions. 


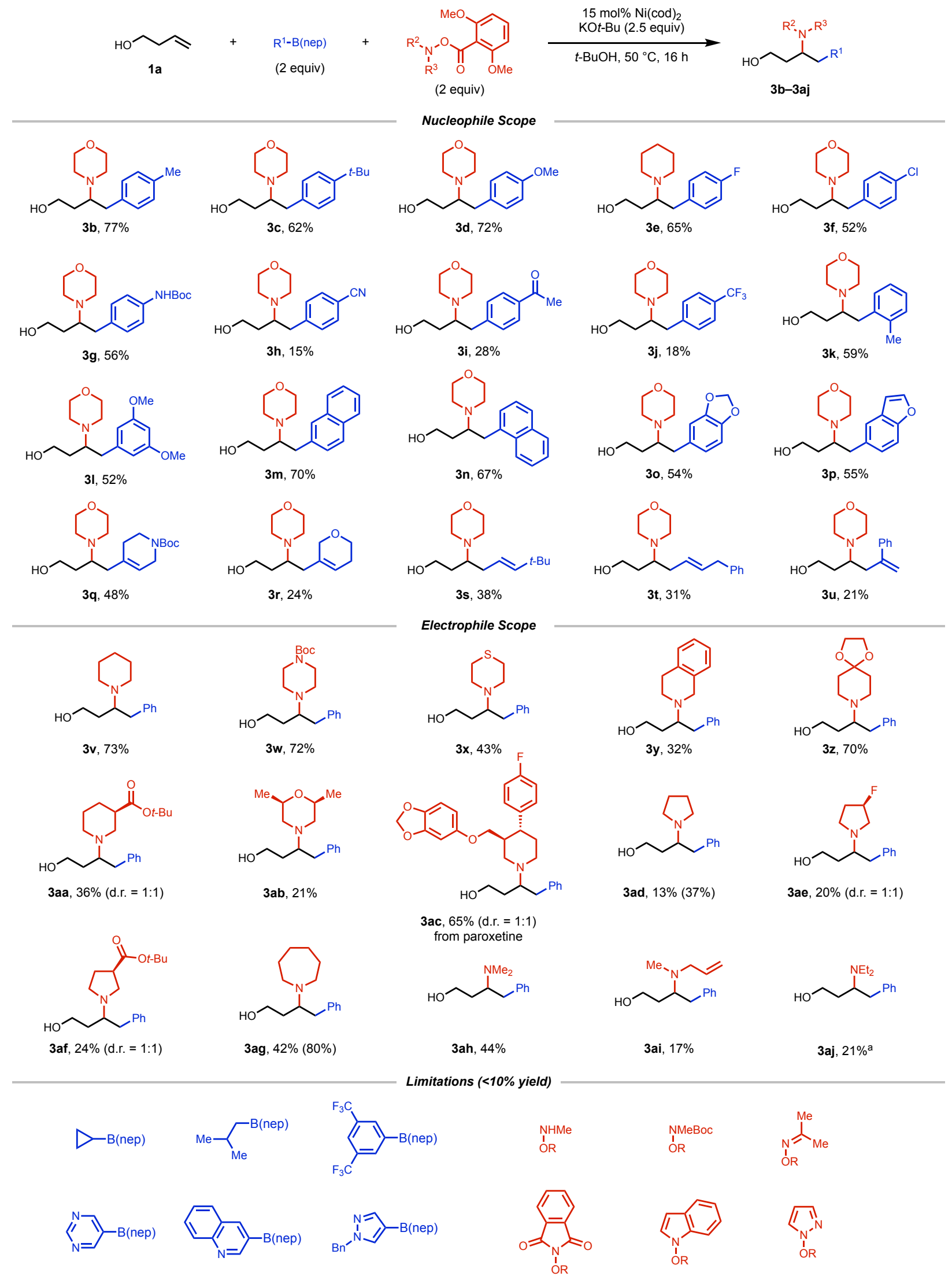


Table 2. Nucleophile and electrophile scope. Reaction conditions: 1a ( $0.1 \mathrm{mmol})$, Aryl/AlkenylB(nep) (0.2 mmol), N-O electrophile (0.2 mmol), KOt-Bu (0.25 mmol), Ni(cod) (0.015 mmol), $t$-BuOH $(1 \mathrm{~mL}), 50{ }^{\circ} \mathrm{C}, 16 \mathrm{~h}$. Percentages represent isolated yields. Values in parenthesis are ${ }^{1} \mathrm{H}$ NMR yields using $\mathrm{CH}_{2} \mathrm{Br}_{2}$ as internal standard. ${ }^{a} N, N$-Diethyl- $O-(2,4,6-$ trimethylbenzoyl)hydroxylamine was used as the electrophile.

\begin{abstract}
Alkene scope. Next, we examined the scope of alkenyl alcohols. The reaction performed well with allyl and bis-homoallyl alcohols (3ak and 3al), demonstrating tolerance for varying chain lengths. Additionally, secondary alcohols (3am-3ao), tertiary alcohols (3ap), and phenol (3aq) were viable native directing groups that deliver the desired products in moderate to excellent yields. We then investigated disubstituted alkene substrates, which pose a formidable challenge compared to terminal alkenes due to the sterically encumbered nature of the migratory insertion step. A representative 1,1-disubstituted alkene was compatible with the reaction conditions (3as), and various 1,2-disubsituted alkenes also delivered the corresponding products with excellent diastereoselectivity, albeit in low yields (3at-3ba). The relative all-cis-stereochemistry of $\mathbf{3 b a}$ was confirmed by single-crystal X-ray diffraction, establishing unambiguously that the reaction is synstereoselective and alcohol directed. In addition to alkenyl alcohol substrates, we demonstrated that our reaction is compatible with sulfonamide directing groups ${ }^{28}$ which can be further deprotected and diversified to give interesting diamine complexes (3bb and $\mathbf{3 b c}$ ). On the other hand, tris-homoallyl alcohol, cyclopentenol, cyclohexenol, and several trisubstituted alkenes were found to be ineffective substrates. Here it is worth noting that all alkenyl alcohol substrates used in table 3 were obtained directly from commercial suppliers, illustrating the widespread availability of this substrate class.
\end{abstract}




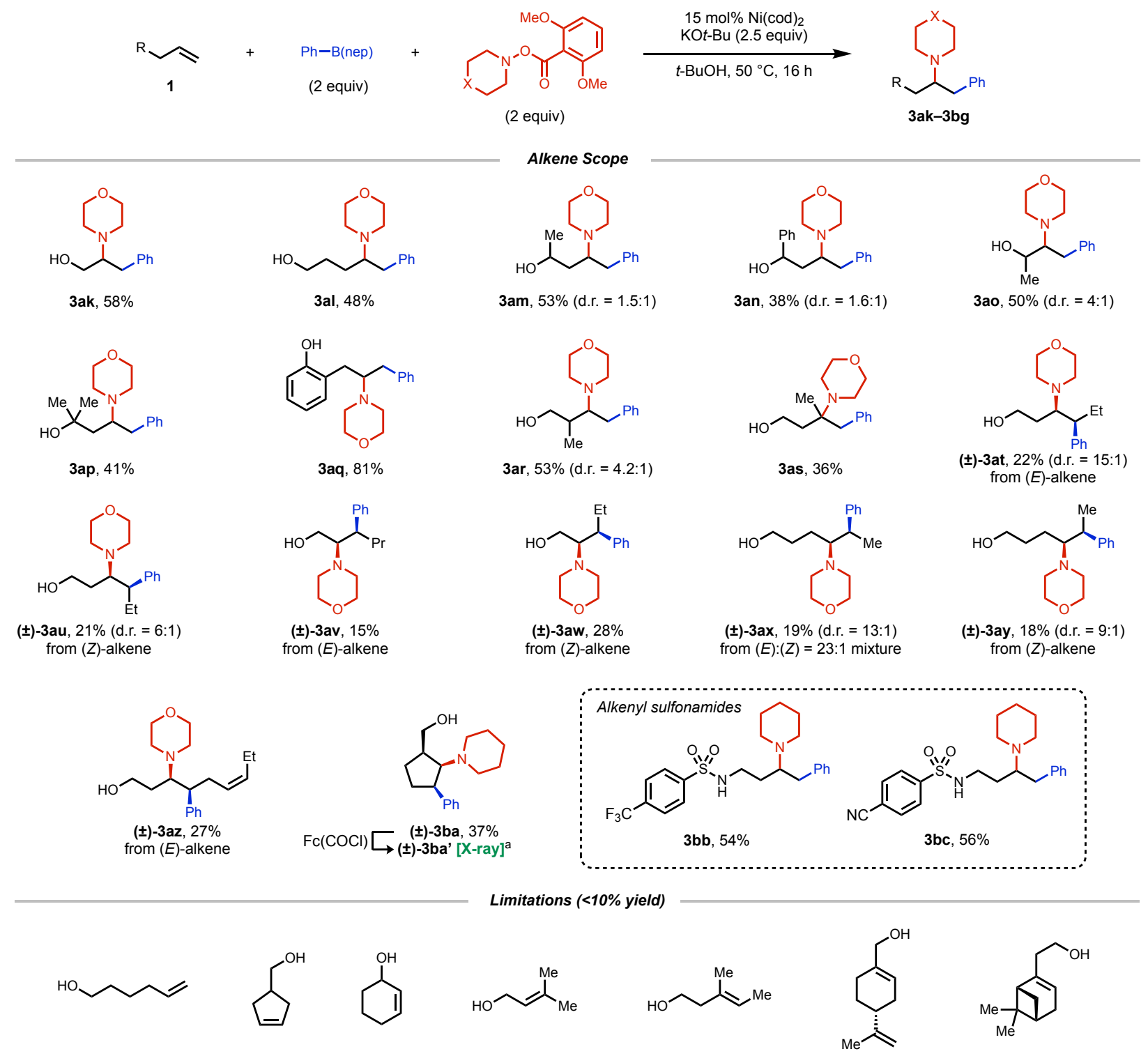

Table 3. Alkene scope. Reaction conditions: 1 ( $0.1 \mathrm{mmol}), \mathrm{PhB}(\mathrm{nep})(0.2 \mathrm{mmol}), \mathrm{N}-\mathrm{O}$ electrophile (0.2 mmol), $\mathrm{KO} t$ - $\mathrm{Bu}(0.25 \mathrm{mmol}), \mathrm{Ni}(\mathrm{cod}){ }_{2}(0.015 \mathrm{mmol}), t-\mathrm{BuOH}(1 \mathrm{~mL}), 50{ }^{\circ} \mathrm{C}, 16$ h. Percentages represent isolated yields. ${ }^{a}$ See SI for X-ray crystallography data.

\section{Large-scale reaction and synthetic application.}


To illustrate the synthetic utility of alcohol-directed 1,2-carboamination, we performed a largescale reaction and showcased several applications to real-world bioactive molecules. Indeed, desired carboaminated product 3w was successfully obtained on 5-mmol scale in good yield. Additionally, two bioactive molecules in the literature were successfully synthesized from the corresponding simple homoallylic alcohol using our developed method followed by functionalization of the versatile hydroxyl group. First, the hydroxyl group of aminoalcohol 3a was triflated in situ and further reacted with an amine nucleophile to give the desired SKP2 inhibitor $\mathbf{6}$ in only two total steps ${ }^{38}$. In addition, the TRPA1 agonist precursor 7 was synthesized in two steps using the presented 1,2-arylamination followed by oxidation of the alcohol to the carboxylic acid ${ }^{39}$. These routes are not only step-economical, but also provide new divergent synthetic pathways that are amenable toward rapid structural diversification for drug discovery.

We next performed the late-stage difunctionalization of complex natural products that contain allylic/homoallylic alcohol moieties. Gratifyingly, linalool, sclareol, allylestrenol, and altrenogest were successfully carboaminated in good to excellent yields (3bd-3bg) without having to install an additional directing auxiliary or any protecting groups.
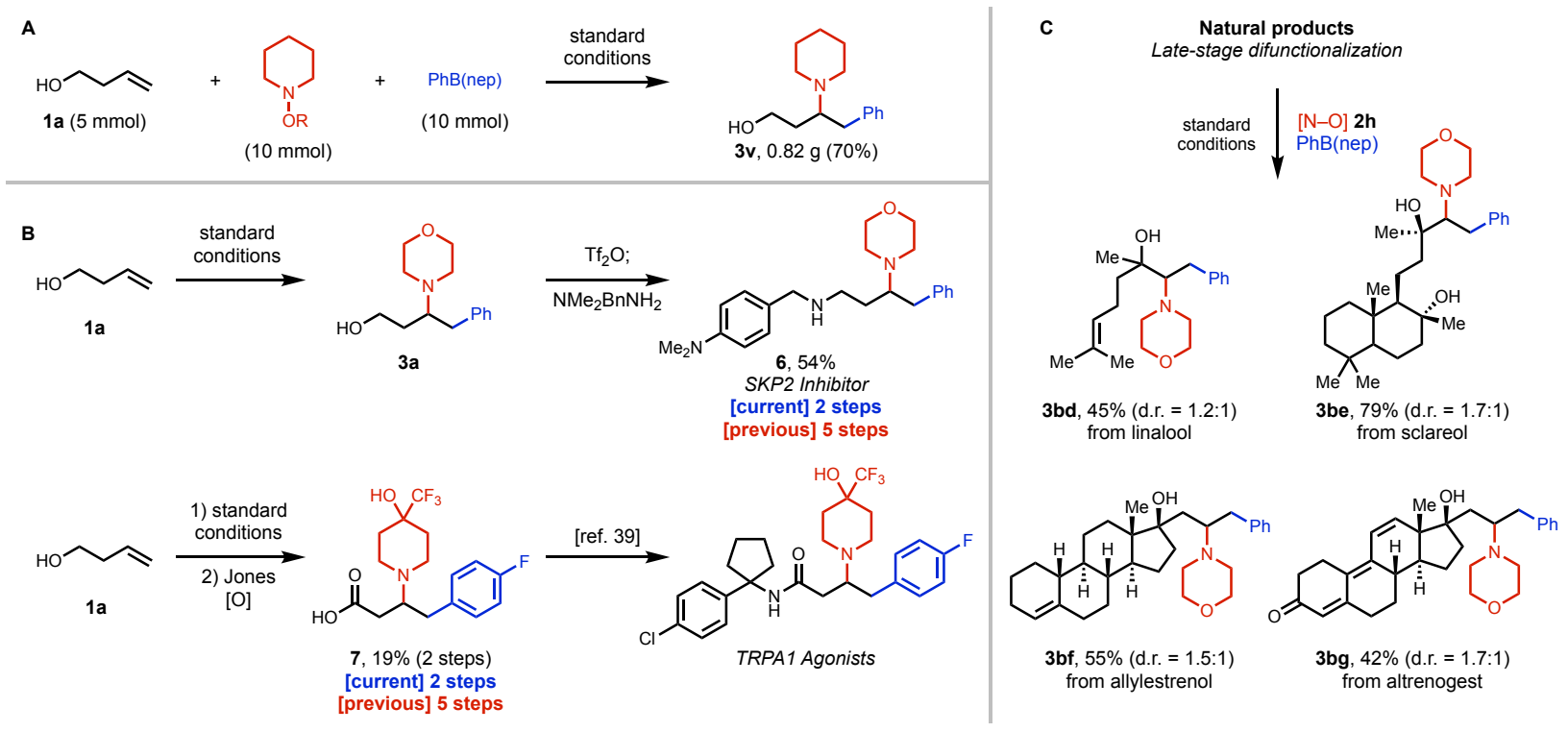
Fig. 2 Large-scale reaction and synthetic application. See SI for details regarding scale and specific conditions.

\section{Mechanistic studies and proposed catalytic cycle.}

The high pathway selectivity of this three-component coupling process and the importance of the tailored $\mathrm{N}-\mathrm{O}$ electrophile prompted us to investigate the reaction mechanism. To this end, we first examined the importance of the alcohol directing group through a series of control experiments. Notably, 4-phenyl-1-butene (8) and a representative alkenyl ether (9), both of which lack an alcohol directing group, resulted in no product formation (Figure 3A). Moreover, Heck or hydroarylated products were not observed in either case, signaling a significant role of the alcohol directing group in the migratory insertion step. We next tested cinnamyl alcohol (10) as a starting material, and in this case, we did not observe any hydroaminated or carboaminated product, with unreacted 10 detected as the major component of the crude reaction mixture (Figure $3 \mathrm{~B}$ ). This result rules out a stepwise mechanism consisting of oxidative Heck arylation followed by hydroamination of the resulting styrenyl intermediate. Finally, a radical clock experiment using 1,6-heptadien-4-ol (11) was performed to probe the generation of a carbon-centered radical intermediate (Figure 3C). Interestingly, acyclic carboaminated product $\mathbf{1 2}$ was observed as the major product, and no evidence of the cyclized product was observed. This result is consistent with a non-radical pathway or alternatively with a radical pathway involving a radical capture rate faster than that of the radical cyclization step $\left(>10^{5} \mathrm{M}^{-1} \mathrm{~s}^{-1}\right){ }^{40}$

With a general picture of the mechanism established, we next considered all of the different possible orders in which the three key elementary steps (TM: transmetalation, OA: oxidative 
addition, and MI: migratory insertion) could take place prior to $\mathrm{C}-\mathrm{N}$ reductive elimination (see SI for details). The three most plausible pathways are: (1) TM-MI-OA, (2) OA-TM-MI, (3) TMOA-MI. To evaluate the viability of these possible sequences, we analyzed the ratio of carboaminated product (3a) to hydroarylated side product (13), the latter of which was obtained in $6 \%$ yield under the standard conditions, as a function of reagent concentrations. In a sequence in which MI precedes OA, both the desired product (3a) and hydroarylated product (13) would be generated from a common alkylnickel(I) intermediate (14); in this case the product ratio should be determined by the relative rates of bimolecular oxidative addition versus protodemetalation (both of which are expected to be irreversible $)^{41}$ and should thus be dependent on the concentration of the $\mathrm{N}-\mathrm{O}$ reagent. In an alternative $\mathrm{OA}-$ first mechanism, the thusly generated alkylnickel(III) intermediate would already bear the amido ligand, and the product ratio should be independent of the $\mathrm{N}-\mathrm{O}$ reagent concentration. Interestingly, we observed that the carboaminated (3a) to hydroarylated (13) product ratio increased when the $\mathrm{N}-\mathrm{O}$ reagent concentration was increased, while alkene concentration does not affect the product ratio (Figure 3D). These data show that the $\mathrm{N}-\mathrm{O}$ reagent, and not the alkene, is involved in the product-determining step, which provides support for pathway (1) the TM-MI-OA sequence.

Based on these experiments and literature precedents, a plausible catalytic cycle is proposed in Figure $3 \mathrm{E}^{19,41,42}$. Here we propose a $\mathrm{Ni}(\mathrm{I}) / \mathrm{Ni}(\mathrm{III})$ catalytic cycle based on the observation of stereospecificity erosion (3at, 3au, 3ax and 3ay), which may imply a competitive alkyl-Ni(III) homolysis/recombination process. The proposed catalytic cycle initiates with formation of an organonickel species via transmetalation of the carbon nucleophile. Next, alcohol-directed syn1,2-migratory insertion occurs to afford a putative alcohol-coordinated alkyl nickelacycle. This 
intermediate then oxidatively adds to the electrophilic aminating reagent and undergoes carbonnitrogen reductive elimination to deliver the 1,2-carboaminated product.

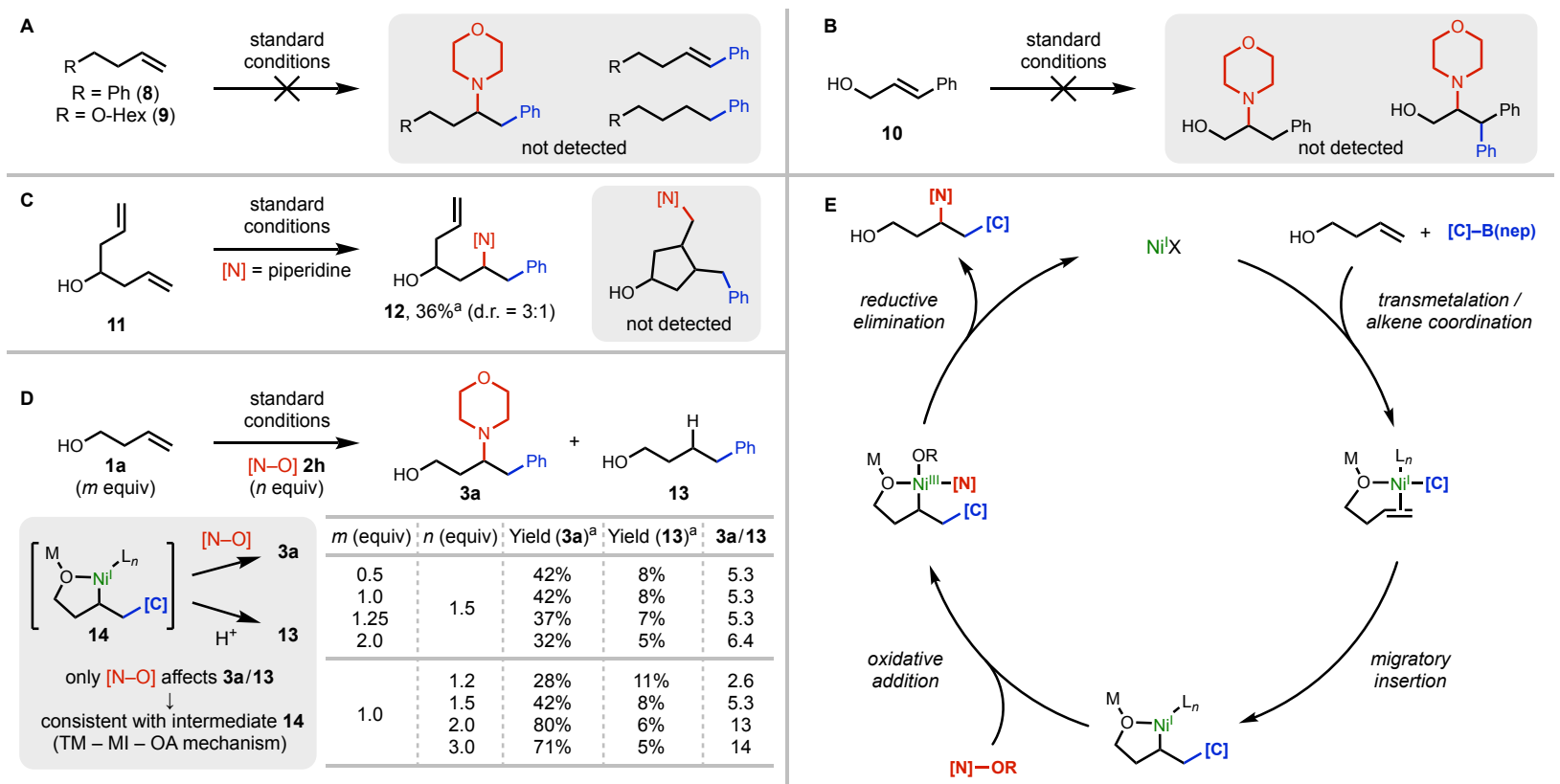

Fig 3. Mechanistic investigation. Summary of mechanistic experiments and proposed catalytic cycle. A. Directing group control experiments. B. Control experiment with potential intermediate. C. Radical clock experiment. D. Substrates loading experiments and product-determining step. E. Proposed catalytic cycle. $\mathrm{M}=\mathrm{K}, \mathrm{H}$, or free lone pair (overall anionic complex). ${ }^{a}$ Percentages represent ${ }^{1} \mathrm{H}$ NMR yields using 1,3,5-trimethoxybenzene as internal standard.

\section{Conclusions}

In summary, we have demonstrated an alcohol-directed, nickel-catalyzed three-component 1,2carboamination of unactivated alkenes. Sterically and electronically modulated $O-(2,6-$ dimethoxybenzoyl)hydroxylamine was found to be a key amine coupling partner for optimal reactivity and minimization of undesired side products. This method was compatible with various 
aryl nucleophiles, alkenyl nucleophiles, and electrophilic amine coupling partners. In addition, diverse alkene substrates including alkenyl alcohols of varying chain lengths, secondary alcohols, tertiary alcohols, disubstituted alkenes, natural products, and sulfonamide-protected alkenyl amines afforded corresponding 1,2-carboaminated products with high regio- and diastereoselectivity. Based on the all-cis-conformation of cyclopentene-derived product 3ba and the results of various control experiments, hydroxyl groups were shown to actively direct chemoand regioselectivity of the desired nickel catalysis. Finally, a gram-scale reaction and applications to construction of bioactive compounds exemplified the synthetic utility of this alcohol-directed alkene 1,2-carboamination.

\section{Data availability}

The data supporting the findings of this study are available within the article and its Supplementary Information.

\section{References:}

1. Henkel, T., Brunne, R. M., Müller, H. \& Reichel, F. Statistical Investigation into the Structural Complementarity of Natural Products and Synthetic Compounds. Angew. Chem., Int. Ed. 38, 643-647 (1999).

2. Vitaku, E., Smith, D. T. \& Njardarson, J. T. Analysis of the Structural Diversity, Substitution Patterns, and Frequency of Nitrogen Heterocycles Among U.S. FDA Approved Pharmaceuticals. J. Med. Chem. 57, 10257-10274 (2014).

3. Garlets, Z. J., White, D. R. \& Wolfe, J. P. Recent Developments in $\mathrm{Pd}^{0}$-Catalyzed AlkeneCarboheterofunctionalization Reactions. Asian J. Org. Chem. 6, 636-653 (2017). 
4. Rosewell, C. F., Sibbald, P. A., Liskin, D. V. \& Michael, F. E. Palladium-Catalyzed Carboamination of Alkenes Promoted by N-Fluorobenzenesulfonimide via $\mathrm{C}-\mathrm{H}$ Activation of Arenes. J. Am. Chem. Soc. 131, 9488-9489 (2009).

5. Faulkner, A., Scott, J. S. \& Bower, J. F. An Umpolung Approach to Alkene Carboamination: Palladium Catalyzed 1,2-Amino-Acylation, -Carboxylation, -Arylation, -Vinylation, and Alkynylation. J. Am. Chem. Soc. 137, 7224-7230 (2015).

6. Yang, H. -B., Pathipati, S. R. \& Selander, N. Nickel-Catalyzed 1,2-Aminoarylation of Oxime Ester-Tethered Alkenes with Boronic Acids. ACS Catal. 7, 8441-8445 (2017).

7. Romanov-Michailidis, F., Sedillo, K. F., Neely, J. M. \& Rovis, T. Expedient Access to 2,3Dihydropyridines from Unsaturated Oximes by $\mathrm{Rh}(\mathrm{III})$-Catalyzed $\mathrm{C}-\mathrm{H}$ Activation. J. Am. Chem. Soc. 137, 8892-8895 (2015).

8. Tasker, S. Z. \& Jamison, T. F. Highly Regioselective Indoline Synthesis under Nickel/Photoredox Dual Catalysis. J. Am. Chem. Soc. 137, 9531-9534 (2015).

9. Zhao, D., Vásquez-Céspedes, S. \& Glorius, F. Rhodium(III)-Catalyzed Cyclative Capture Approach to Diverse 1-Aminoindoline Derivatives at Room Temperature. Angew. Chem., Int. Ed. 54, 1657-1661 (2015).

10. Lee, S., Semakul, N. \& Rovis, T. Direct Regio- and Diastereoselective Synthesis of $\delta$-Lactams from Acrylamides and Unactivated Alkenes Initiated by $\mathrm{Rh}^{\mathrm{III}}$-Catalyzed $\mathrm{C}-\mathrm{H}$ Activation. Angew. Chem., Int. Ed. 59, 4965-4969 (2020).

11. Ni, H. -Q., Kevlishvili, I., Bedekar, P. G., Barber, J. S., Yang, S., Tran-Dubé, M., Romine, A. M., Lu, H. -X., McAlpine, I. J., Liu, P. \& Engle, K. M. Anti-selective [3+2] (Hetero)annulation 
of Non-conjugated Alkenes via Directed Nucleopalladation. Nat. Commun. 11, 6432-6439 (2020).

12. Piou, T. \& Rovis, T. Rhodium-catalysed syn-carboamination of alkenes via a transient directing group. Nature 527, 86-90 (2015).

13. Huang, H. -M., Koy, M., Serrano, E., Pflüger, P. M., Schwarz, J. L. \& Glorius, F. Catalytic radical generation of $\pi$-allylpalladium complexes. Nat. Catal. 3, 393-400 (2020).

14. Jiang, H. \& Studer, A. Intermolecular Radical Carboamination of Alkenes. Chem. Soc. Rev. 49, 1790-1811 (2020).

15. Pinkert, T., Wegner, T., Mondal, S. \& Glorius, F. Angew. Chem., Int. Ed. 58, 15041-15045 (2019).

16. Li, Z., Zhang, M., Zhang, Y., Liu, S., Zhao, J. \& Zhang, Q. Multicomponent Cyclopropane Synthesis Enabled by $\mathrm{Cu}$-Catalyzed Cyclopropene Carbometalation with Organoboron Reagent: Enantioselective Modular Access to Polysubstituted 2-Arylcyclopropylamines. Org. Lett. 21, 5432-5437 (2019).

17. Zhang, Y., Li, Y., Zhou, W., Zhang, M., Zhang, Q., Jia, R. \& Zhao, J. Assembly of Polysubstituted Chiral Cyclopropylamines via Highly Enantioselective Cu-Catalyzed ThreeComponent Cyclopropene Alkenylamination. Chem. Commun. 56, 12250-12253 (2020).

18. Liu, Z., Wang, Y., Wang, Z., Zeng, T., Liu, P. \& Engle, K. M. Catalytic Intermolecular Carboamination of Unactivated Alkenes via Directed Aminopalladation. J. Am. Chem. Soc. 139, 11261-11270 (2017).

19. van der Puyl, V. A., Derosa, J. \& Engle, K. M. Directed, Nickel-Catalyzed Umpolung 1,2Carboamination of Alkenyl Carbonyl Compounds. ACS Catal. 9, 224-229 (2019). 
20. Yanagisawa, A., Nomura, N., Habaue, S. \& Yamamoto, H. Nickel-Catalyzed Regioselective Allylation of Allylic Alcohols. Tetrahedron Lett. 30, 6409-6412 (1989).

21. Crabtree, R. H. Homogeneous Transition Metal Catalysis of Acceptorless Dehydrogenative Alcohol Oxidation: Applications in Hydrogen Storage and to Heterocycle Synthesis. Chem. Rev. 117, 9228-9246 (2017).

22. Hoveyda, A. H., Evans, D. A. \& Fu, G. C. Substrate-Directable Chemical Reactions. Chem. Rev. 93, 1307-1370 (1993).

23. Bhadra, S. \& Yamamoto, H. Substrate Directed Asymmetric Reactions. Chem. Rev. 118, 3391-3446 (2018).

24. Zhang, J.-S., Liu, L., Chen, T. \& Han, L.-B. Transition-Metal-Catalyzed Three-Component Difunctionalization of Alkenes. Chem. Asian J. 13, 2277-2291 (2018).

25. Wang, Z.-X., Bai, X.-Y. \& Li, B.-J. Metal-Catalyzed Substrate-Directed Enantioselective Functionalization of Unactivated Alkenes. Chin. J. Chem. 37, 1171-1180 (2019).

26. Liu, Z., Zeng, T., Yang, K. S. \& Engle, K. M. $\beta, \gamma$-Vicinal Dicarbofunctionalization of Alkenyl Carbonyl Compounds via Directed Nucleopalladation. J. Am. Chem. Soc. 138, 15122-15125 (2016).

27. Derosa, J., Kleinmans, R., Tran, V. T., Karunananda, M. K., Wisniewski, S. R., Eastgate, M. D. \& Engle, K. M. Nickel-Catalyzed 1,2-Diarylation of Simple Alkenyl Amides. J. Am. Chem. Soc. 140, 17878-17883 (2018).

28. Apolinar, O., Tran, V. T., Kim, N., Schmidt, M. A., Derosa, J. \& Engle, K. M. Sufonamide Directivity Enables Ni-Catalyzed 1,2-Diarylation of Diverse Alkenyl Amines. ACS Catal. 10, $14234-14239(2020)$. 
29. Xi, Y. \& Hartwig, J. F. Diverse Asymmetric Hydrofunctionalization of Aliphatic Internal Alkenes through Catalytic Regioselective Hydroboration. J. Am. Chem. Soc. 138, 6703-6706 (2016).

30. Derosa, J., Kang, T., Tran, V. T., Wisniewski, S. R., Karunananda, M. K., Jankins, T. C., Xu, K. L. \& Engle, K. M. Nickel-Catalyzed 1,2-Diarylation of Alkenyl Carboxylates A Gateway to 1,2,3-Trifunctionalized Building Blocks. Angew. Chem. Int. Ed. 59, 1201-1205 (2020).

31. Rousseau, G. \& Breit, B. Removable Directing Groups in Organic Synthesis and Catalysis. Angew. Chem. Int. Ed. 50, 2450-2494 (2011).

32. Katsuki, T. \& Sharpless, K. B. The First Practical Method for Asymmetric Epoxidation. J. Am. Chem. Soc. 102, 5974-5976 (1980).

33. Sawano, T. \& Yamamoto, H. Substrate-Directed Catalytic Selective Chemical Reactions. $J$. Org. Chem. 83, 4889-4904 (2018).

34. Hu, D. X., Shibuya, G. M. \& Burns, N. Z. Catalytic Enantioselective Dibromination of Allylic Alcohols. J. Am. Chem. Soc. 135, 12960-12963 (2013).

35. Hu, D. X., Seidl, F. J., Bucher, C. \& Burns, N. Z. Catalytic Chemo-, Regio, and Enantioselective Bromochlorination of Allylic Alcohols. J. Am. Chem. Soc. 137, 3795-3798 (2015).

36. Seidl, F. J., Min, C., Lopez, J. A., Burns, N. Z. Catalytic Regio- and Enantioselective Haloazidation of Allylic Alcohols. J. Am. Chem. Soc. 140, 15646-15650 (2018).

37. Bandar, J. S., Pirnot, M. T. \& Buchwald, S. L. Mechanistic Studies Lead to Dramatically Improved Reaction Conditions for the Cu-Catalyzed Asymmetric Hydroamination of Olefins. J. Am. Chem. Soc. 137, 14812-14818 (2015). 
38. Shouksmith, A. E., Evans, L. E., Tweddle, D. A., Miller, D. C., Willmore, E., Newell, D. R., Golding, B. T. \& Griffin, R. J. Synthesis and Activity of Putative Small-Molecule Inhibitors of the F-Box Protein SKP2. Aust. J. Chem. 68, 660-679 (2015).

39. Chernov-Rogan, T., Gianti, E., Liu, C., Villemure, E., Cridland, A. P., Hu, X., Ballini, E., Lange, W., Deisemann, H., Li, T., Ward, S. I., Hackos, D. H., Magnuson, S., Safina, B., Klein, M. L., Volgraf, M., Carnevale, V. \& Chen, J. TRPA1 Modulation by Piperidine Carboxamides Suggests an Evolutionarily Conserved Binding Site and Gating Mechanism. Proc. Natl. Acad. Sci. U. S. A. 116, 26008-26019 (2019).

40. Newcomb, M. in Encyclopedia of Radicals in Chemistry, Biology and Materials, Vol 1 (eds Chatgilialoglu, C. \& Studer, A.) (John Wiley \& Sons, Weinheim, 2012).

41. Jeon, J., Lee, C., Seo, J. \& Hong, S. NiH-Catalyzed Proximal-Selective Hydroamination of Unactivated Alkenes. J. Am. Chem. Soc. 142, 20470-20480 (2020).

42. He, J., Xue, Y., Han, B., Zhang, C., Wang, Y. \& Zhu, S. Nickel-Catalyzed Asymmetric Reductive 1,2-carboamination of Unactivated Alkenes. Angew. Chem. Int. Ed. 59, 2328-2332 (2020).

Supplementary Information is linked to the online version of the paper at www.nature.com/nature.

Acknowledgments: We thank Omar Apolinar, Van T. Tran and Camille Z. Rubel for detailed proofreading of this manuscript and supporting information and Dr. Joseph Derosa (Caltech) for helpful discussion. We further thank Dr. Milan Gembicky (USCD) for assistance with X-ray 
crystallographic analysis. Dr. Jason Chen, Brittany Sanchez, and Emily Sturgell (Scripps Research Automated Synthesis Facility) are acknowledged for HRMS analysis.

Funding: This work was financially supported by Bristol Myers Squibb, the National Science Foundation (CHE-1800280), and the Camille Dreyfus Teacher-Scholar Program. We further acknowledge the Kwanjeong Educational Foundation for a Graduate Fellowship (T.K.).

\section{Affiliations}

${ }^{1}$ Department of Chemistry, The Scripps Research Institute, 10550 North Torrey Pines Road, La Jolla, CA 92037, United States.

${ }^{2}$ Discovery Chemistry, Bristol Myers Squibb, PO Box 4000, Princeton, NJ 08543, United States.

\section{Author contributions}

T.K. and K.M.E. conceived the project. T.K. optimized the reaction conditions. T.K., N.K., P.T.C., H.Z., and K.F. examined the scope of the transformation. T.K. performed mechanistic experiments. P.T.C. and K.M.E. directed the research. T.K. and K.M.E. wrote the manuscript with input from all authors.

\section{Competing interests}

The authors declare no competing interests.

\section{Corresponding author}

Correspondence to: keary@scripps.edu 